\title{
Investigación científica o investigación jurídica en las facultades de Derecho de las universidades licenciadas en Perú*
}

\author{
Scientific research or legal research in the faculties of Law of the LIcensed universities in Peru \\ A pesquisa científica ou pesquisa jurídica nas faculdades de direito das universidades licenciadas no Perú
}

DOI: https://doi.org/10.21803/pensam.v17i21-1.273

Rubén Walter Huaranga Soto https://orcid.org/0000-0003-3644-3594

\section{¿Cómo citar este artículo?}

Huaranga, R. (2018). Investigación Científica - Investigación Jurídica en las Facultades de Derecho de las Universidades Licenciadas en Perú. Pensamiento Americano, 11(22), 156-167. DOI: https://doi.org/10.21803/pensam.v17121-1.273

\begin{abstract}
Resumen
La investigación en las Facultades de Derecho de las Universidades Licenciadas continúan con el uso de la Metodología de la Investigación Jurídica que no es otra forma de aceptar y desarrollar la Metodología de la Investigación científica, con ello se reduce al estudio del campo del Derecho sólo al ámbito dogmático jurídico; por lo que realizar la investigación con uno u otro método limita la posibilidad de alcanzar conocimientos que nos permitan precisar qué es el Derecho y como es que se manifiesta en una sociedad de estado de derecho en la que son las normas que buscan cambiary mejorar situaciones problemáticas. Si se continua bajo un modelo único de metodología de investigación, no se logrará avanzar en el campo del Derecho, por lo que es necesario que las universidades permitan que la investigación se realice a partir de las diferentes metodologías tanto de carácter filosófico, sociológico entre otras posibilidades tal como se puede encontrar en las diversas producciones bibliográficas sobre el conocimiento del Derecho; por ello se propone la implementación de una investigación metodológica abierta frente a las limitaciones que se encuentra establecido en los planes de estudios en las facultades de Derecho de las universidades licenciadas.
\end{abstract}

Palabras Clave: Investigación en derecho; metodología de investigación

\begin{abstract}
Research in the Law Faculties of the Licensed Universities continues with the use of the Legal Research Methodology which is not another way of accepting and developing the Methodology of Scientific Research, thereby reducing it to the study of the field of law only by dogmatic legal field. Therefore, carrying out research with one or another method limits the possibility of reaching knowledge that allows us to specify what the law is and how it manifests itself in a society of the rule of law in which are the norms that seek to change and improve situations problematic If it is continued under a unique model of research methodology. It will not be possible to advance in the field of law, so it is necessary that universities allow research to be carried out based on the different methodologies both philosophical, sociological, among others. Possibilities as can be found in the various bibliographical productions on the knowledge of law; therefore, the implementation of an open methodological investigation is proposed in the face of the limitations established in the curricula in the law schools of the licensed universities.
\end{abstract}

Key words: Research in law, Research methodology.

\footnotetext{
* Derivación de la ponencia. Investigación en proceso.
} 


\section{Resumo}

A pesquisa nas Faculdades de Direito das Universidades Licenciadas continua com o uso da Metodologia de Pesquisa Jurídica que não é outra forma de aceitar e desenvolver tal metodologia, com isso se reduz o estudo de campo do Direito somente em âmbito dogmático jurídico; assim realizar a pesquisa com um ou outro método limita a possibilidade de alcançar conhecimentos que nos permitam precisar o que é o Direito e como é que este se manifesta numa sociedade de Estado de Direito no qual são as normas que buscam mudar e melhorar as situações problemáticas. Ao permanecer baixo um modelo único de metodologia em pesquisa não se avançará no campo do Direito, pelo qual é necessário que as universidades permitam que a pesquisa se realize a partir de diferentes metodologias tanto de caráter filosófico, sociológico, entre outras possibilidades tal como se encontra em diversas produções bibliográficas sobre o conhecimento do Direito. Por isso, se propõe a implementação de uma pesquisa metodológica aberta frente às limitações que se encontre estabelecido nos planos de estudo das faculdades de Direito das universidades licenciadas.

Palavras Chave: Pesquisa em Direito, metodologias de pesquisa

\section{Perfil}

Defensor Universitario, Docente de la Facultad de Derecho y Ciencias Políticas, Docente de Posgrado Walter.huaranga@untrm. edu.pe.warangas@hotmail.com

Rubén Walter Huaranga Soto

Magister en Derecho Civil y Comercial 


\section{Introducción}

ntes de la nueva ley universitaria, no
se efectuaba ni se exigía una tesis
para titularse; la mayoría de los egresados recurría a la defensa de dos expedientes judiciales; en el mejor de los casos, quién ejecutaba una Tesis recurría a la Metodología de la Investigación Científica o Metodología de la Investigación Jurídica. Hoy, se promueve la investigación; aunque mantiene la posibilidad de titulación por otras modalidades que cada universidad podría establecer. No obstante, para la obtención del grado de bachiller, los egresados universitarios tienen que presentar un trabajo de investigación. En relación con los libros de investigación en el campo del Derecho; en su mayoría recurre a la Metodología de la Investigación Científica, bajo el título de Investigación Jurídica o Metodología en la Investigación en Derecho. Conviene subrayar que no se han realizado investigaciones sobre estos manuales o textos de investigación; de ahí que es importante establecer que metodología o metodologías de investigación se deben utilizar en el Derecho.

La nueva ley Universitaria $N^{\circ} 30220$, publicada el 08 de julio de 2014; establece que las universidades nacionales y privadas obtengan el licenciamiento; procedimiento de carácter obligatorio para la continuación del servicio que brindan las universidades; para ello deben tener las condiciones básicas de calidad, entre ellas, la infraestructura y equipamiento adecuados, líneas de investigación a desarrollarse, contar con no menos del $25 \%$ de docentes calificados a tiempo completo. Crea el Vicerrectorado de Investigación. En dicha Ley se encuentra, la palabra investigación por 72 oportunidades en los 133 artículos y las 24 disposiciones complementarias, transitorias, modificatorias, finales y derogatorias de la norma; con lo cual se colige el interés en la producción de conocimiento y que las universidades sean las que fortalezcan esta función.

De otro lado, encontramos que los docentes de investigación de las Facultades de Derecho son sociólogos o licenciados en educación o ingenieros. Asimismo, en los planes de estudio de las Facultades de Derecho, no se presta atención al rubro investigación ni se cuenta con las condiciones mínimas para realizar investigación en el campo del Derecho.

El presente trabajo se realiza sobre las universidades licenciadas; dado que son las que se han adecuado a la ley universitaria, supuestamente de acuerdo con las exigencias que se requiere para el funcionamiento de estas; además porque cuentan con un currículo y el énfasis en la investigación. A partir de ello, se presenta como alternativa "Los Métodos de Investigación en Derecho". Lo que permitiría que las Facultades de Derecho promuevan la investigación y de ese modo se contribuya al conocimiento en este campo. Ya que, a pesar de ello, no le prestan atención a la investigación; se continúa con la formación profesional tradicional; tal es así que las asignaturas orientadas a la investigación son muy pocas. A pesar de ello, encontramos universidades que cuentan con la edición de revistas sobre Derecho.

Por lo tanto, el propósito de este artículo es incentivar la discusión y adhesión a los que propugnan la necesidad de investigar el derecho en forma libre, más allá del dogmatismo jurídico; dadas la condiciones que nos brinda la Ley universitaria 30220; centrada en la investigación y no solo en la formación de profesionales eficientitas.

\section{Desarrollo de la temática}

Las formas de acercamiento al conocimien- 
to de la naturaleza, de la sociedad y del pensamiento son cada vez más profundas; cada día se descubre nuevas cosas que generan admiración y a la vez incertidumbres sobre si ya llegamos a conocer los hechos y fenómenos que logramos percibirlos y es así que encontramos una gran cantidad de trabajos de investigación, sobre todo orientados al desarrollo de la tecnología en desmedro de la propia ciencia, en especial de las ciencias sociales y culturales; tal es el caso que las investigaciones sobre el Derecho, se han limitado al estudio de la norma jurídica, de sus efectos en la aplicación de solución de controversias o en el castigo a quienes atentan contra la sociedad; es más, nos hemos alejado del entorno inmediato, a lo que llamamos realidad; los trabajos de investigación se realizan sobre hechos del pasado; mientras que la expectativa es tener estudios de carácter prospectivo; de tal modo que el Derecho cobre importancia en el desarrollo de una sociedad de bienestar, seguridad y con justicia.

Cuando hablamos de la realidad, nos referimos a la conciencia que tenemos de las cosas que hacemos o que suceden permitiéndonos no sólo adecuarnos y soportarlos; sino que podemos enfrentarlos y modificarlos; incluso establecer una forma de realidad a partir de comprensión. Es así como Bedoya (2017), expresa "Así, nuestra realidad no es otra cosa que una construcción que hacemos con el lenguaje y que como puede resultar ya claro, no se reduce a "nombrar" el mundo, sino a proporcionarnos una forma especial de relación con él: conocerlo" (p.28). De modo que podamos aportar con los cambios que requiera la sociedad.

El Derecho como norma jurídica, no es estático ni evoluciona, simplemente es distinto en cada época. Tal es así que Broekman (1997), dice que "el derecho occidental moderno está basado en la idea fundamental del contrato" (p.17). Por consiguiente, desde este punto de vista el derecho es contractual, circunstancial, irrepetible, fenece y sólo es válido para las partes bajo circunstancias temporales y locales. Esta forma de reducir el Derecho a una norma y su insistencia en su estudio es la que nos paraliza y nos mantiene en el pasado, en lo que sucedió, en buscar las causas de hechos jurídicos que necesitan atenderse, de tal modo que cuando se da solución, se da solución a un hecho pasado y a una supuesta prevención, es decir a que no se vuelva a cometer actos que afecten la sociedad.

Al mismo tiempo, se observa que:

Los hechos jurídicos son construidos por los juristas, son el resultado de su actividad discursiva específica. En cambio, los hechos de la realidad cotidiana no son construidos de esta manera estricta, ligada al lenguaje y a las formas del derecho (Broekman, 1997, p.23).

$\mathrm{Si}$, se quieren conocer estos hechos como objeto del derecho, no se debe limitar el método de estudio a una única forma de investigar, ya que:

Explicar ese "todo" que se insinúa en lo que decimos resulta literalmente imposible; sólo lograr imaginar la cantidad de construcciones de "realidad" que han permitido que todo ello ocurra o se produzca, es ya un esfuerzo que puede quedar desmedido para una buena mayoría de nosotros (Bedoya, 2017, p.97).

El problema radica en que "Los teóricos del derecho no suelen estar pendientes expresamente de los cambios en los métodos de las ciencias naturales: ese no es campo de su estudio" (Martínez, 2012, p.62). Los científicos han pasado de la certeza a la probabilidad y

Pensamiento Americano Vol. 11 (22) • 2018 • Julio-Diciembre • Corporación Universitaria Americana • Barranquilla, Colombia • ISSN: $2027-2448$. 
es más la incertidumbre, como parte de los resultados que obtienen al tratar de exponer resultados de cada investigación. De igual modo, tenemos que los avances en las ciencias empíricas se desarrollan en la propuesta intensa de la física cuántica, en observar aquello que no se puede percibir sensorialmente sin ayuda de instrumentos y aun así sus resultados siempre se encontrarán en algo circunstancial, transitorio.

Si éstos son los cambios en los que nos encontramos en la construcción del conocimiento; cuando se busca resolver una controversia jurídica podemos decir que: "Al alejarse la ciencia de las certidumbres, al no ser su objetivo prioritario el ofrecerlas, el Derecho no puede encontrar en ella un apoyo firme para fundar sus decisiones" (Esteve \& Tejada, 2013, p.57). Por lo tanto, no se tendría la certeza en las decisiones jurídicas.

\section{Como lo menciona Carpintero (2018):}

Los datos precedentes del mundo de la mecánica actual apenas han afectado a la visión aristotélica del hombre... No parece que haya algún inconveniente propiamente científico para declarar que la visión aristotélica del hombre puede ser considerada una metafísica natural gracias a la que el hombre logra entenderse a sí mismo más acabadamente que no de la mano de otras propuestas sobre ética (p. 93).

En tal sentido, se puede aceptar la necesidad de volver a Aristóteles para conocer al hombre como ser social, al derecho como manifestación de la convivencia social y cultural, la ética como parte de convivencia y fuente de reglas que orienten una mejor y acertada elaboración de aceptación de las diferencias y diversidad de percepciones y construcciones del conocimiento.
Los llamados a generar investigación en derecho, desde diversas metodologías y posibilidades, son las Facultades de Derecho, en base no sólo a las políticas estatales; sino que como tarea y objeto de ser las universidades las generadoras de conocimientos. En tal sentido es necesario que se deje libertad metodológica en la construcción de nuevos conocimientos y nuevas formas de acercamiento al Derecho.

El estudio del derecho ha sido atrapado por el método de investigación científica, método que nos permite conocer sólo una arista del derecho, la norma jurídica; con ello ha quedado estático el avance de conocer qué es el derecho ni para qué es el derecho. Se acusa al medioevo como la más oscurantista en el desarrollo del conocimiento; sin embargo, hoy en día pretender observar y conocer el mundo con una sola perspectiva es mucho más limitante; en tal sentido "cada tipo de realidades tiene su acceso epistemológico propio, y que cada cosa solamente puede ser conocida en la medida en que ella se deja conocer" (Martínez, 2012, p.40).

La vigencia, popularidad y exigencia de seguir el modelo de la metodología de la investigación científica se basa en el hecho de una sociedad economicista; en ese sentido Esteve y Tejada (2013), dicen:

En una sociedad que ha fiado su futuro, su continuidad y su bienestar al progreso científico y técnico... El reto que se plantea es cómo afrontar estas cuestiones, de gran impacto social en muchos casos, y decidir sobre ellas, cuando las instancias jurídicas y políticas constitucionalmente habilitadas para decidir carecen de conocimiento para ello... (p.10).

Las otras ciencias sociales y otras formas culturales de entender al ser humano como ser

Pensamiento Americano Vol. 11 (22) • 2018 • Julio-Diciembre • Corporación Universitaria Americana • Barranquilla, Colombia • ISSN: $2027-2448$. 
social y con mayor razón los profesionales de Derecho debemos asumir la necesidad de promover investigaciones para presentar alternativas a la mejora de condiciones de vida de las diferentes sociedades culturales a partir de nuestra propia realidad y praxis cotidiana inmediata y prospectiva.

Como alternativa a la problemática de llegar a conocer el Derecho, consideramos que debemos tener en cuenta la pluriculturalidad, la interdisciplinariedad y la temporalidad histórica en donde se manifiesta el fenómeno; para ello la diversidad de métodos de investigación deben incorporarse en la formación de abogados; de tal modo que se propicie en los egresados de la Facultades de Derecho de las universidades licenciadas, contar con juristas y no sólo con abogados procesalistas dogmáticos. Sousa y Meneses (2016), "Toda experiencia social produce y reproduce conocimiento $\mathrm{y}$, al hacerlo, presupone una o varias epistemologías" (p.7). En tal sentido, la búsqueda de conocimiento no puede darse por una sola vía, un método monopólico; se necesita una serie de caminos que lleven a un acercamiento a ese Derecho para conocerlo en sus diferentes aristas, más allá de la norma jurídica.

Consideramos la importancia de:

... qué es lo que debemos estudiar, en qué aspectos nos debemos centrar cuando, al menos a primera vista, el derecho presenta múltiples dimensiones, por lo que siempre está latente la duda de a dónde debemos acudir, en qué debemos centrar la atención y cómo debemos proceder para conocer qué derechos tenemos nosotros o las personas que esperan esa respuesta de nosotros (Martínez, 2012, p.11).

Sobre todo, la necesidad de contar con diversas metodologías de investigación y de la interdisciplinariedad como necesidad de llegar a describir, explicar y comprender qué es el Derecho. Puesto que hasta antes y después del positivismo los estudiosos del derecho siempre han utilizado diversas formas de estudio; por lo que las universidades son las llamadas, a través de sus Facultades de Derecho, a promover la investigación en el campo del Derecho en una forma abierta y libre de formas únicas de métodos de investigación. Entre los aspectos importantes, mas no se han tocado, son la filosofía del derecho que nos permitirá tener una visión como punto de partida de la comprensión del derecho; la epistemología como disciplina que en vez de establecer formas únicas de desarrollar el conocimiento, debe permitir el reconocimiento de epistemologías diversas; por último, el tema de la política estatal en el reforzamiento de la investigación integral, interdisciplinaria, libre de dogmatismos y no sólo promover la investigación tecnológica repetitiva.

\section{Cuestiones metodológicas}

Antes de nada, se ha revisado textos sobre el conocimiento del Derecho y su relación con la ciencia; se ha revisado la Ley Universitaria vigente sobre las exigencias de licenciamiento y sobre las formas de obtener la titulación; luego se ha recurrido a la indagación del número de universidades licenciadas, de los cuales fueron seleccionados aquellas que cuentan con Facultades de Derecho y Ciencias Políticas. Se recurrido a los planes de estudios de donde se obtiene el número de cursos de investigación por semestres y por la carrera. Se extrajo información del Registro Nacional de Investigadores en Ciencia y Tecnología para conocer el número de docentes investigadores abogados de las universidades; de igual modo a páginas web relacionadas a dichas universidades para recopilar información sobre los planes de estudios y publicaciones.

Pensamiento Americano Vol. 11 (22) · 2018 • Julio-Diciembre · Corporación Universitaria Americana • Barranquilla, Colombia • ISSN: $2027-2448$. 
Tabla 1.

Matriz de universidades licenciadas con facultades de Derecho y cursos de investigación incorporados en los planos de estudios.

UNIVER-

CICLOS

TOTAL

\begin{tabular}{|c|c|c|c|c|c|c|c|c|c|c|c|c|}
\hline \multirow{2}{*}{ SIDAD } & \\
\hline & $\mathbf{I}$ & II & III & IV & $\mathbf{V}$ & VI & VII & VIII & IX & $\mathbf{X}$ & XI & XII \\
\hline U.N. & Met. &.-- &.-- & Inv. & Met. de &.-- & Met. de & Inv. & Proyect & Desarro &.-- & Informe de \\
\hline Toribio & del & & & Docum & la Inv. & & la Inv. & Docume & o de & 1lo de la & & Inv. \\
\hline Rodríguez & Trabajo & & & ental I & Científi & & Jurídica & ntal II & Inv. & Inv. & & \\
\hline de & Univers & & & & $\mathrm{ca}$ & & & & & & & \\
\hline Mendoza & itario & & & & & & & & & & & \\
\hline \multicolumn{13}{|l|}{ de } \\
\hline \multicolumn{13}{|l|}{ Amazonas } \\
\hline U. Nacional &.-- &.-- &.-- &.-- &.-- &.-- &.-- &.-- &.-- & Met. de & Met. de & Met. de la \\
\hline del & & & & & & & & & & la Inv. & la Inv. & Inv. Jurídica \\
\hline \multirow[t]{4}{*}{ Altiplano } & & & & & & & & & & Jurídica & Jurídica & III (Informe \\
\hline & & & & & & & & & & I (Proy. & II & y Redacción \\
\hline & & & & & & & & & & De & (Ejecuc & Científica) \\
\hline & & & & & & & & & & Inv.) & ión) & \\
\hline
\end{tabular}

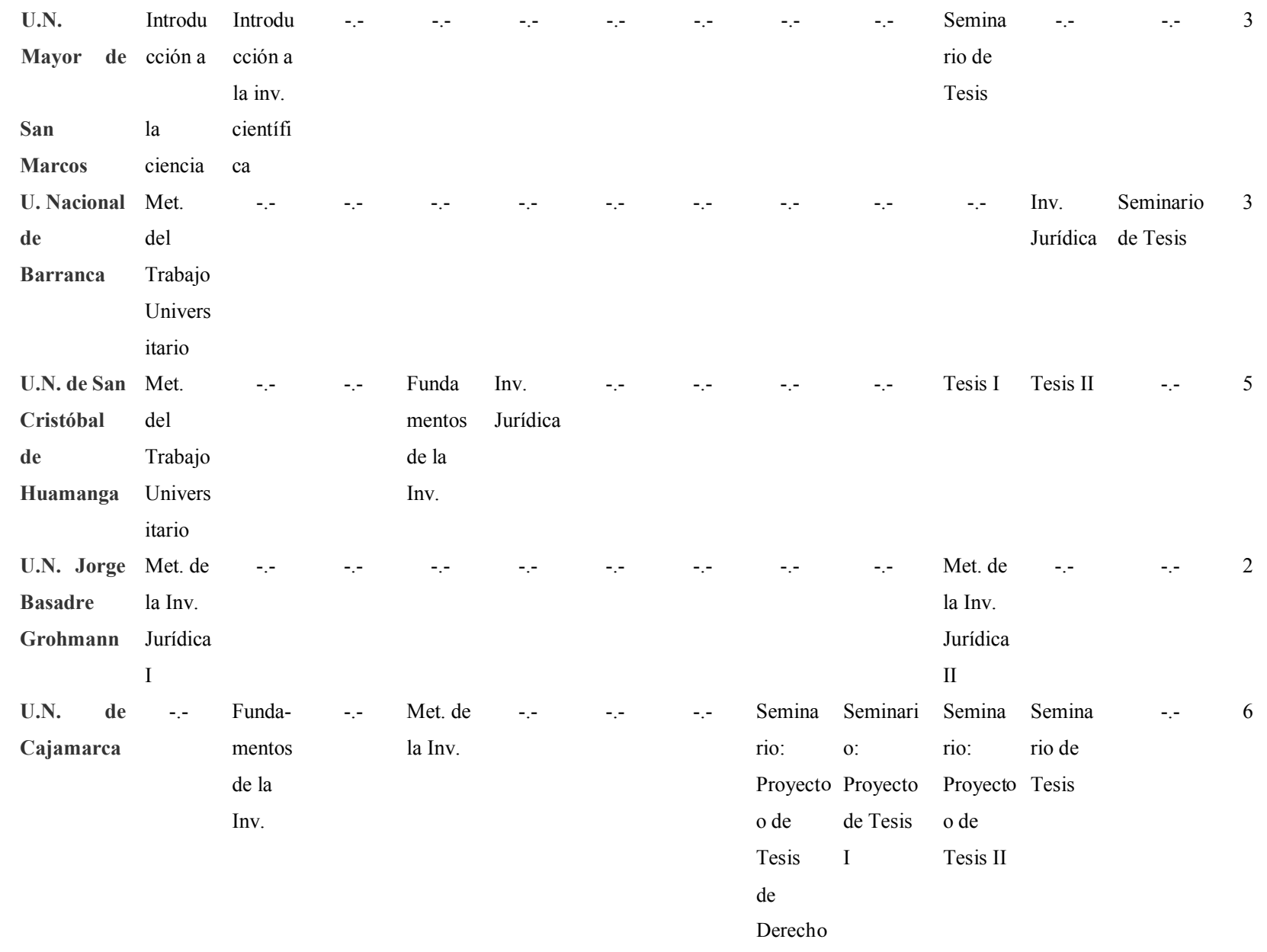




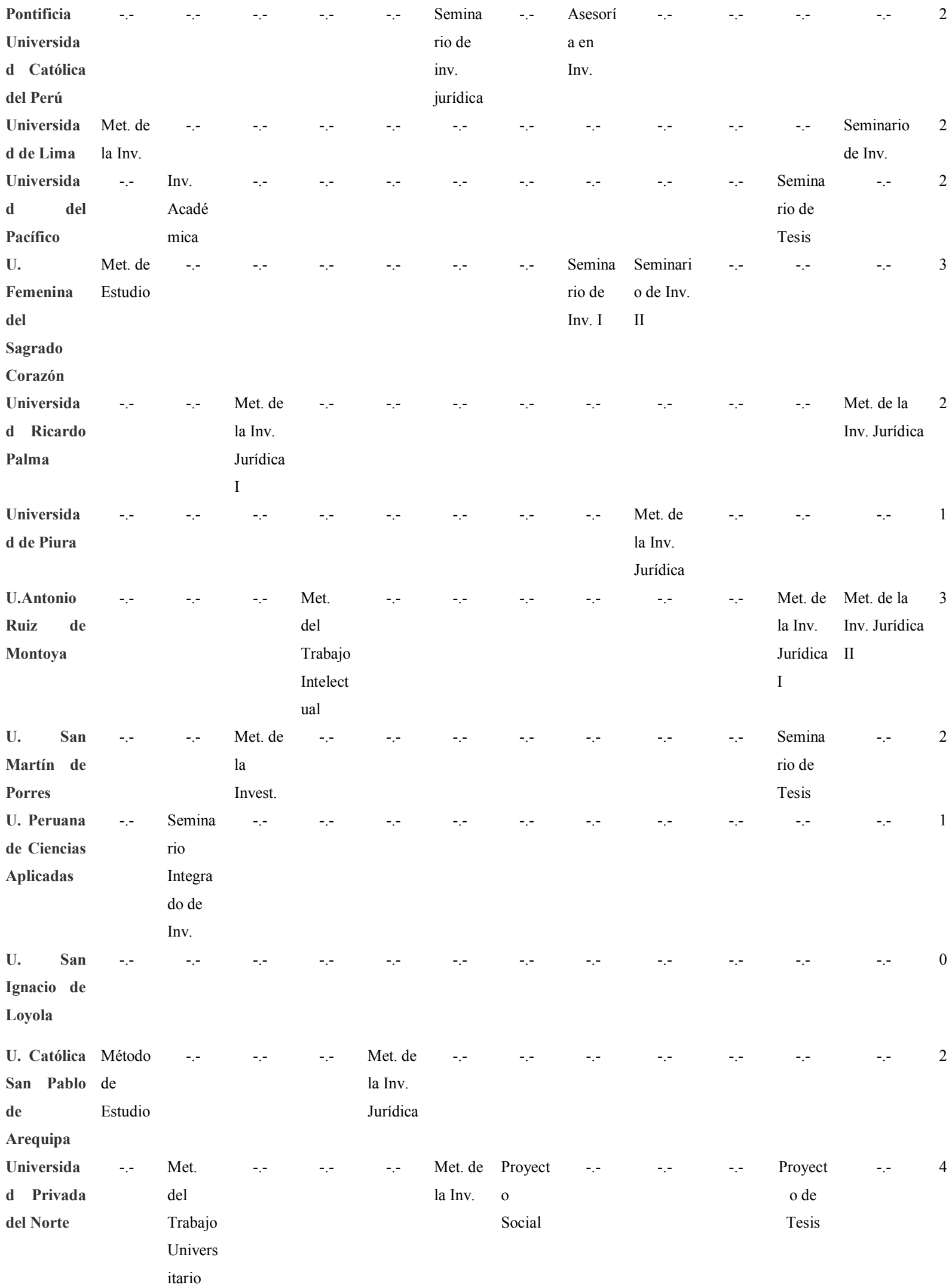




\begin{tabular}{|c|c|c|c|c|c|c|c|c|c|c|c|c|c|}
\hline $\begin{array}{l}\text { Universida } \\
\text { d Privada } \\
\text { de Tacna }\end{array}$ & $\begin{array}{l}\text { Met. } \\
\text { del } \\
\text { Trabajo } \\
\text { Univers } \\
\text { itario }\end{array}$ & -.- & -.- &.-- &.-- &.-- &.-- &.-- &.-- &.-- & $\begin{array}{l}\text { Semina } \\
\text { rio de } \\
\text { Tesis I }\end{array}$ & $\begin{array}{l}\text { Seminario } \\
\text { de Tesis II }\end{array}$ & 3 \\
\hline $\begin{array}{l}\text { Universida } \\
\text { d Andina } \\
\text { del Cusco }\end{array}$ & $\begin{array}{l}\text { Taller } \\
\text { de } \\
\text { Método } \\
\text { s de } \\
\text { Estudio } \\
\text { Univers } \\
\text { itario }\end{array}$ & -.- &.-- &.-- & -.- &.-- &.-- &.-- &.-- & $\begin{array}{l}\text { Met. de } \\
\text { la Inv. }\end{array}$ & $\begin{array}{l}\text { Semina } \\
\text { rio o } \\
\text { Taller } \\
\text { de } \\
\text { Tesis I }\end{array}$ & $\begin{array}{l}\text { Seminario o } \\
\text { Taller de } \\
\text { Tesis II }\end{array}$ & 4 \\
\hline $\begin{array}{l}\text { U. Católica } \\
\text { de Santa } \\
\text { María }\end{array}$ &.-- & $\begin{array}{l}\text { Met. } \\
\text { del } \\
\text { Trabajo } \\
\text { Univers } \\
\text { itario }\end{array}$ &.-- &.-- &.-- &.-- &.-- &.-- &.-- &.-- & $\begin{array}{l}\text { Met. de } \\
\text { la Inv. } \\
\text { Jurídica }\end{array}$ & $\begin{array}{l}\text { Taller de } \\
\text { Inv. Jurídica }\end{array}$ & 3 \\
\hline $\begin{array}{l}\text { Universida } \\
\text { d La Salle }\end{array}$ &.-- &.-- & -.- &.-- & $\begin{array}{l}\text { Met. de } \\
\text { la Inv. } \\
\text { Científi } \\
\text { ca }\end{array}$ & -.- &.-- &.-- & -.- & $-\cdot-$ & $\begin{array}{l}\text { Semina } \\
\text { rio de } \\
\text { Inv. I }\end{array}$ & $\begin{array}{l}\text { Seminario } \\
\text { de Inv. II }\end{array}$ & 3 \\
\hline $\begin{array}{l}\text { U.Católica } \\
\text { Santo } \\
\text { Toribio de } \\
\text { Mogrovejo }\end{array}$ & $\begin{array}{l}\text { Met. } \\
\text { del } \\
\text { Trabajo } \\
\text { Intelect } \\
\text { ual }\end{array}$ & $\begin{array}{l}\text { Met. de } \\
\text { la Inv. }\end{array}$ & -.- &.-- & -.- &.-- &.-- &.-- & -.- & $\begin{array}{l}\text { Met. de } \\
\text { la Inv. }\end{array}$ & $\begin{array}{l}\text { Taller } \\
\text { de } \\
\text { Titulaci } \\
\text { ón I }\end{array}$ & $\begin{array}{l}\text { Taller de } \\
\text { Titulación } \\
\text { II }\end{array}$ & 5 \\
\hline $\begin{array}{l}\text { U.Privada } \\
\text { Antenor } \\
\text { Orrego }\end{array}$ & $\begin{array}{l}\text { Met. } \\
\text { del } \\
\text { Trabajo } \\
\text { Univers } \\
\text { itario }\end{array}$ & -.- & -.- &.-- & $\begin{array}{l}\text { Met. de } \\
\text { la Inv. }\end{array}$ & -.- &.-- &.-- & -.- & $\begin{array}{l}\text { Proyect } \\
\text { o de } \\
\text { Inv. } \\
\text { científi } \\
\text { ca }\end{array}$ & Tesis I & Tesis II & 5 \\
\hline $\begin{array}{l}\text { Universida } \\
\text { d ESAN }\end{array}$ & & & & & & $\begin{array}{l}\text { Met. de } \\
\text { la Inv. }\end{array}$ & & & & $\begin{array}{l}\text { Asesorí } \\
\text { a de } \\
\text { Tesis I }\end{array}$ & $\begin{array}{l}\text { Asesorí } \\
\text { a de } \\
\text { Tesis II }\end{array}$ & & 3 \\
\hline $\begin{array}{l}\text { Universida } \\
\text { d Científica } \\
\text { del Sur }\end{array}$ & & & $\begin{array}{l}\text { Met. de } \\
\text { la Inv. } \\
\text { Para } \\
\text { Humani } \\
\text { dades }\end{array}$ & & & & & & $\begin{array}{l}\text { Seminari } \\
\text { o de Inv. } \\
\text { Jurídica }\end{array}$ & $\begin{array}{l}\text { Semina } \\
\text { rio de } \\
\text { Tesis }\end{array}$ & & & 3 \\
\hline $\begin{array}{l}\text { Universida } \\
\text { d } \\
\text { Continenta } \\
\text { l }\end{array}$ &.-- & -.- & -.- &.-- & -.- & $\begin{array}{l}\text { Semina } \\
\text { rio de } \\
\text { Inv. }\end{array}$ &.-- &.-- &.-- & -.- & $\begin{array}{l}\text { Taller } \\
\text { de Inv. } \\
1 \\
\text { Derech } \\
\text { o }\end{array}$ & $\begin{array}{l}\text { Taller de } \\
\text { Inv. } 2 \\
\text { Derecho }\end{array}$ & 3 \\
\hline 28 & 12 & 7 & 3 & 4 & 5 & 4 & 2 & 4 & 5 & 11 & 12 & 13 & 86 \\
\hline
\end{tabular}

Fuente: Elaboración del autor. 
En cuanto a la forma de investigación en Derecho, se ha podido visualizar que la metodología de la investigación científica es la forma mono-metodológica de investigar; lo que conlleva a una investigación jurídica, la cual estudia apenas una de las aristas del Derecho.

\section{Resultados}

Se ha constatado el número limitado de cursos de investigación en los planes de estudios de las Facultades de Derecho de las universidades licenciadas.

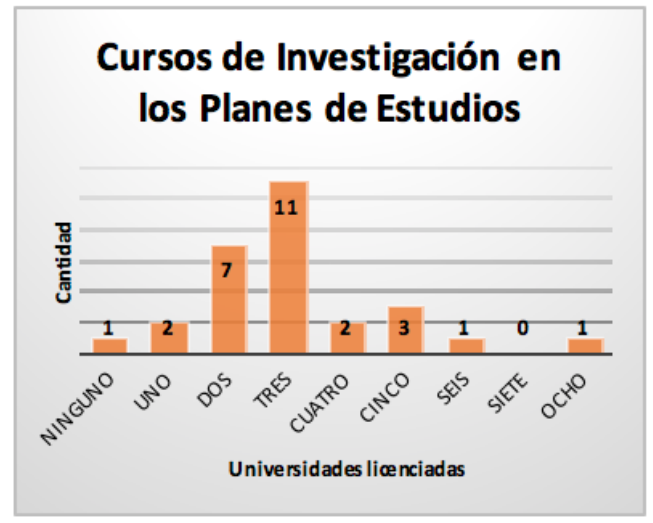

Gráfica 1: Cantidad de cursos de investigación en la formación de abogados por Facultades de Derecho.

Fuente: Elaboración del autor.

La ubicación y distribución de cursos se concentran en los tres últimos ciclos; además que como curso propedéutico de investigación uno en el primer ciclo.

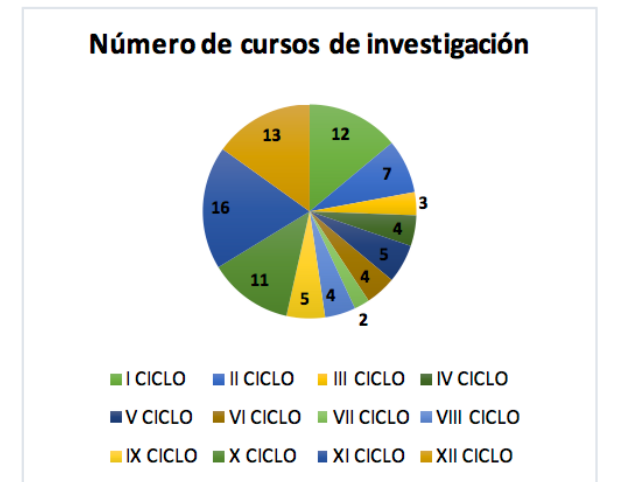

Gráfica 2: Cantidad de cursos en las Facultades de Derecho. Fuente: Elaboración del autor.
Después de revisar la página de REGINA, Se encontró un número reducido de docentes abogados registrados como investigadores.

Tabla 2.

Distribución de frecuencias de docentes abogados de 28 universidades licenciadas reconocidos como investigadores por REGINA.

\begin{tabular}{llc}
\hline \multicolumn{1}{c}{ Universidades } & \multicolumn{2}{c}{ Docentes } \\
\cline { 2 - 3 } Universidad Nacional & $\mathrm{f}$ & $\mathrm{h}$ \\
\cline { 2 - 3 } Mayor de San Marcos & 1 & 3,6 \\
Universidad de San & 2 & 7,1 \\
Martin de Porras & & \\
Universidad de Lima & 1 & 3,6 \\
Universidad del & 1 & 3,6 \\
Pacifico & & \\
Universidad de Piura & 2 & 7,1 \\
Otras 23 sin docentes & 0 & 75,0 \\
investigadores & & 100,0 \\
REGINA & 7 & \\
\hline \multicolumn{2}{c}{28} &
\end{tabular}

Fuente: Elaboración del autor.

De acuerdo con la búsqueda realizada en Latindex (2018), se han encontrado que vigentes un total de seis revistas de Derecho; Derecho y Foro Jurídico de la Pontificia Universidad Católica del Perú; Revista de Derecho de la Universidad Católica de Santa María; Revista de Derecho de la Universidad de Piura; IUS de la Universidad Católica Santo Toribio de Mogrovejo e IUS Inkarri de la Universidad Ricardo Palma.

Se elaboró un gráfico como síntesis de las metodologías de investigación en Derecho que deberían de ser implementadas como cursos de investigación progresiva; sin dejar de lado la metodología de investigación científica ni la metodología de la investigación jurídica. 


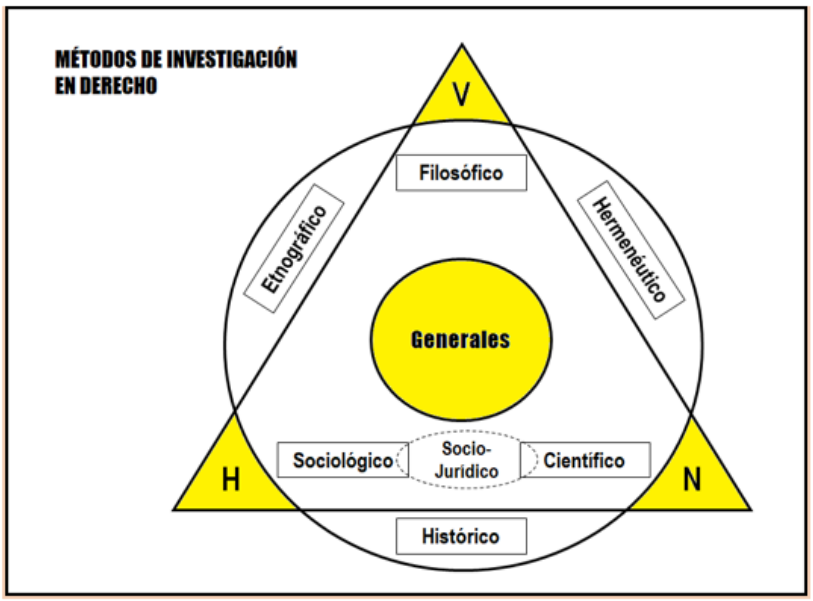

Gráfica 3: Métodos de Investigación en Derecho.

Fuente: Elaboración propia en base a la información teórica consultada.

Dichas metodologías, de ser implementadas en la formación de abogados conllevaría a cumplir con uno de los fines de la universidad, el de generar conocimientos, sobre el Derecho, a partir de nuestra realidad cultural y social.

\section{Discusión}

En la construcción del marco conceptual, para la comprensión de la necesidad de generar conocimiento en el campo del Derecho, se ha definido que se necesita de una forma de estudio del Derecho desde diversas metodologías de acercamiento para poder avanzar en la precisión de qué es el Derecho.

Los límites que establece la nueva ley universitaria y la política nacional de concentrar sus esfuerzos en la ciencia y la tecnología, dejan de lado la importancia del desarrollo del Derecho que de uno u otro modo es parte del desarrollo que se requiere no sólo en las ciencias sino en las diversas formas de interrelación y búsqueda de una sociedad que atienda sus necesidades de bienestar.

El reducido número de cursos, además con contenidos metodológicos limitados a la investigación científica, impide que se siga desarrollando el Derecho; tal es así que los docentes que enseñan investigación son de otras carreras. Si se incentivara la formación de investigadores en derecho y se promocionara la actividad investigativa en el campo social y en especial del Derecho se obtendría propuestas que permitan mejorar la interrelación y convivencia en la sociedad.

Se ha dejado de lado, la necesidad de partir de una epistemología alternativa, la misma que podría llevarnos a precisar las formas de implementación de cada uno de los métodos a usar en la investigación del Derecho. Una de las propuestas la encontraríamos en las "Epistemologías del Sur" o la "Epistemología de la Descolonización" propuestos por Boaventura De Sousa Santos y por Enrique Dussel respectivamente.

\section{Conclusiones}

Se logró establecer que la metodología de investigación científica o la metodología de la investigación jurídica son la misma metodología; Así como el número reducido de cursos de investigación en las Facultades de Derecho de las universidades licenciadas; asimismo se encontró sólo siete docentes abogados registrados como investigadores en REGINA.

Se alcanzó a entender que la Nueva Ley Universitaria aparentemente promueve la investigación al crear los vicerrectorados de investigación, la promoción de docentes investigadores; sin embargo, establece alternativas de titulación distintas a la realización de investigación.

Se propone en forma gráfica las metodologías y las formas de investigación interdisciplinaria de investigación en el campo del Derecho; las mismas que se compila de estudios de otros autores que advierten la ne- 
cesidad de una investigación más abierta y libre en Derecho.

Si la política de Estado en el campo de la investigación fuera universal y no solamente focalizado en la tecnología, entonces la sociedad posibilitaría un mejor manejo de los recursos con que cuenta para mejorar las condiciones de vida.

\section{Referencias}

Bedoya, H. (2017). La construcción epistémica del derecho. Bogotá, Colombia: Universidad Externado de Colombia.

Broekman, J. (1997). Derecho, filosofía del Derecho y Teoría del Derecho. Bogotá, Colombia: Editorial Temis S.A.

Carpintero, B. (2018). El método del Derecho en el cambio científico del Siglo XXI. Madrid, España: Editorial Dykinson.

Congreso de la República de Perú. (9 de julio de 2014)., por medio de la cual establece la creación, funcionamiento, supervisión y cierre de universidades; además promueve la calidad educativa como entes de desarrollo nacional, de la investigación y de la cultura. [Ley No 30220].

Esteve, J. \& Tejada, J. (2013). Ciencia y Derecho: La nueva división de poderes. Madrid, España: Fundación Coloquio Jurídico Europeo.

Latindex. (2018). Características de calidad del catálogo histórico 2002-2017 (revistas en línea). Recuperado de: https://www.latindex.org/latindex/docuElectHist

Martínez, J. (2012). El conocimiento jurídico. (3ª edición). Madrid, España: Servicio de Publicaciones de la Facultad de Derecho de la Universidad Complutense Madrid.

Sousa, B. \& Meneses, M. (2016). Epistemologías del Sur (Perspectivas). España: Ediciones Akal, S.A.

Tribunal constitucional de Perú. (2015). Sentencia de los expedientes 0014-2014-PI/TC, 0016-2014-PI/TC, 00192014-PI/TC y 0007-2015-PI/TC de 2015. Lima. permission: www.americana.edu.co

https://www.coruniamericana.edu.co/publicaciones/ojs/index.php/pensamientoamericano/index 